\title{
Parameter Identification of Nonlinear System on Combustion Engine Based MVEM using PEM
}

\author{
Trigas Badmianto ${ }^{1}$, Eka Firmansyah ${ }^{2}$, Adha Imam Cahyadi ${ }^{3}$
}

\begin{abstract}
In four-stroke engine injection system, often called spark ignition (SI) engine, the air-fuel ratio (AFR) is taken from the measurement of lambda sensor in the exhaust. This sensor does not directly describe how much AFR in the combustion chamber due to the large transport delay. Therefore, the lambda sensor is used only as a feedback in AFR control "correction", not as the "main" control. The purpose of this research is to identify the parameters of the non-linear system in SI engines to produce AFR estimator. The AFR estimator is expected to be used as a feedback of the main "AFR" control system. The process of identifying the parameters using the Gauss-Newton method, due to its rapid computation to Achieve convergence, is based on prediction error minimization (PEM). The models of AFR estimator is an open-loop system without a universal exhaust gas oxygen (UEGO) sensors as feedback, called a virtual AFR sensor. The high price of UEGO sensors makes the virtual AFR sensor can be a practical solution to be applied in AFR control. The model in this research is based on the mean value engine models (MVEM) with some modifications. The research dataset was taken from a Hyundai Verna 2002 with the additional UEGO type of lambda sensors. The throttle opening angle (input) is played by stepping on the gas pedal and the signal to the injector (input) is set to a certain quantity to produce the AFR (output) value read by the UEGO sensor. This research produces an open loop estimator model or AFR virtual sensors with normalized root mean square error (NRMSE) = $0.06831=6.831 \%$.
\end{abstract}

Keywords--Parameter identification, Air-Fuel Ratio, MVEM, Spark-ignition engines, fuel injection system, Prediction error minimization

\section{NOMENCLATURE}

EKF Extended Kalman filter

CGEKF Constant gain extended Kalman filter

EGO Exhaust gas oxygen

BEGO Binary exhaust gas oxygen

UEGO Universal exhaust gas oxygen

AFR Air-fuel ratio

PEM Prediction error minimization

RMSE Root mean square error

NRMSE Normalized root mean square error

TPS Throttle position sensor

MAP Manifold absolut pressure

\footnotetext{
${ }^{1}$ Student, Department of Electrical Engineering and Information Technology, Universitas Gadjah Mada, Jl. Grafika 2, Yogyakarta 55281, Indonesia (tlp: 0274-552305; e-mail: trigas.badmianto@mail.ugm.ac.id)

2, 3 Lecturer, Department of Electrical Engineering and Information Technology, Universitas Gadjah Mada, Jl. Grafika 2, Yogyakarta 55281, Indonesia (tlp: 0274-552305; e-mail: eka.firmansyah@ugm.ac.id,adha.imam@ugm.ac.id)
}

$\begin{array}{ll}\text { ECT } & \begin{array}{l}\text { Engine coolant temperature } \\ \text { Air mass flow rate into cylinder (in kilogram per }\end{array} \\ \dot{m}_{a p} & \begin{array}{l}\text { second) } \\ \text { Air mass flow rate past throttle plate (in kilogram per }\end{array} \\ \dot{m}_{a t} & \text { second) } \\ P_{a} & \text { Atmosphere pressure (1.013 bar) } \\ P_{i} & \text { Manifold air pressure (in bar) } \\ T_{a} & \text { Atmosphere temperature (in Kelvin) } \\ T_{i} & \text { Manifold air temperature (in Kelvin) } \\ n & \text { Crank shaft speed (in kilo revolution per minute) } \\ \dot{m}_{f} & \text { Cylinder port fuel flow (in kilogram per second) } \\ & \text { Fraction of } \dot{m}_{f i} \text { which is deposited on manifold as fuel } \\ X_{f} & \text { film } \\ \tau_{f} & \text { Fuel evaporation time constant (0.25 s) } \\ \dot{m}_{f f} & \text { Fuel film mass flow (in kilogram per second) } \\ \dot{m}_{f v} & \text { Fuel vapor mass flow (in kilogram per second) } \\ \dot{m}_{f i} & \text { Injected fuel mass flow (in kilogram per second) } \\ \lambda & \text { Normalized air-fuel ratio } \\ \alpha & \text { Throttle opening angle (in degree) } \\ e V & \text { Volumetric efficiency }\end{array}$

\section{INTRODUCTION}

Identification of parameters (model) is required to obtain an accurate mathematical model. A good model is a model that more accurately reflects the dynamics of the system or plant. The dynamics of air-fuel ratio (AFR) on SI injection system Engine is very non-linear and multivariable [1], makes the process of the parameter identification difficult compared with the linear system and a few variables.

The AFR is an important indicator that affects exhaust emissions, engine power, and fuel consumption in Spark Ignition (SI) Engine. The influence of lambda (normalized AFR) on power and fuel consumption are presented in Fig. 1. Maximum power will be achieved if the lambda value is in the range of 0.82 (AFR $=12: 1$ ) [2], while the fuel consumption will be achieved if the minimum number lambda is in the range of 1:09 $(\mathrm{AFR}=16: 1)$ [2].

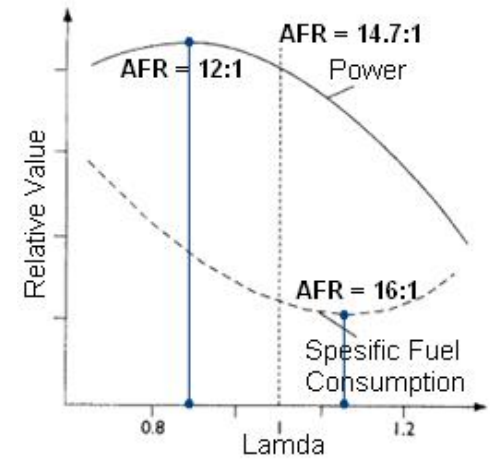

Fig. 1 Graph of power and fuel consumption to changes in the SI lambda engine [2]. 
To reduce emissions, accurate AFR control is required to be directed to ideal conditions (stoichiometric), which is a theoretical figure of 14.7:1 [2], with worth lambda $(\lambda)=1$. AFR value is obtained by reading the lambda sensor or exhaust gas oxygen (EGO) located in the exhaust. In ideal conditions (stoichiometric), the voltage generated by the EGO sensor is about 400 millivolts [2].

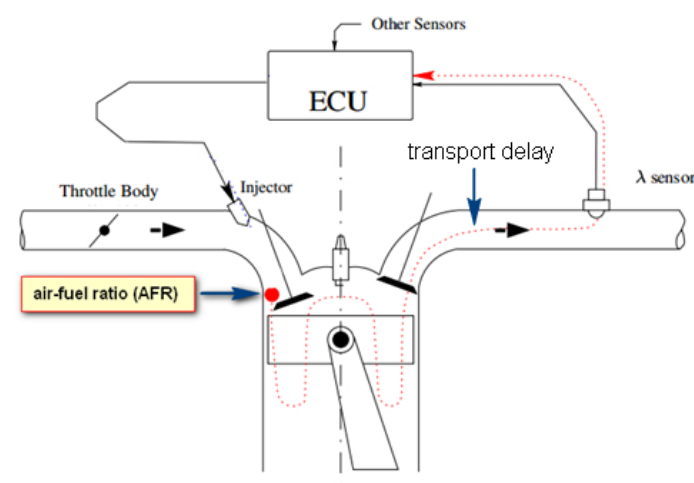

Fig. 2 Delay of AFR on SI engine injection system [3].

In the SI engine injection control system, the EGO sensor output values read by an electronic control unit (ECU) do not directly describe the condition of the AFR in the cylinder (combustion chamber) at the same time, because of the influence of transport delay which is around $250 \mathrm{~ms}$ [4]. This transport delay is affected by the dynamics of the process of mixing air and fuel in the combustion chamber, the length of duct, the exhaust, and the dynamics of the EGO sensor itself as presented in Fig. 2.

Computing in ECU still uses the controller as part of the classical form of injection control system. This classic controller uses algorithms if-then-rule, in the form of look-up table [5], [6], or fuzzy logic controller [5], [7], as feedforward control.

Control the "main" AFR generates a control signal which is dominant to make the injector ON curing conditions of transient and steady state. Transient conditions occur during acceleration or deceleration caused by changes in the opening angle of the throttle a sudden. While the condition steady state occurs when the opening angle throttle that produces a constant output, plant AFR, and engine speed (rpm) constant. The control signal "corrective" plays to increase or decrease the control signal "main" to obtain a difference small between the outputs of AFR and AFR targets. The size of control signal "correction" is approximately $10 \%$ of the control signal "main".

In a classical control at transient conditions, there is the big transport delay on the sensor EGO leading to the control signal "correction" to be late to respond every change in the AFR, so that the sensor EGO is served as the control "correction" AFR, not as a control "main" AFR as shown in Fig. 3 [5], [6].

Logically, by using AFR values in the combustion chamber as feedback, the system controls the "main" can be designed to stand alone as presented by the AFR control system as presented in Fig. 4.

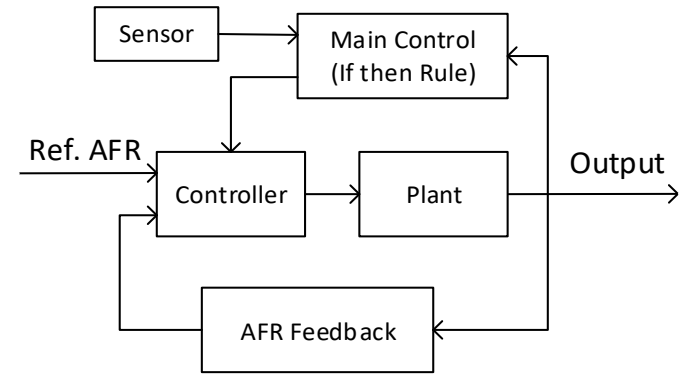

Fig. 3 AFR control system using feedback as a correction.

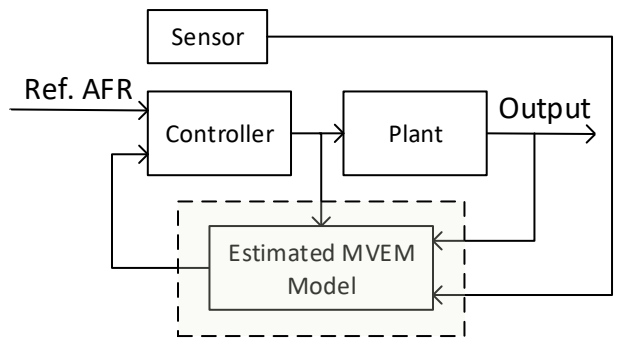

Fig. 4 The main controls in the control AFR system using sensor of estimation results.

However, until now there is no sensor to measure the AFR directly in the combustion chamber with pressurized combustion chamber shape and has a high temperature. Therefore, the way offered in this paper is to estimate the AFR in the combustion chamber (without the influence of transport delay) to an equation of state that is not measured directly by the sensor, but it can be modelled into a mathematical form so that the value of AFR will still be estimated.

There are two ways to estimate the state AFR, the first is to use a UEGO sensor for feedback, and the second is without using UEGO sensor which is also known as virtual AFR sensor. The high price of UEGO type sensor makes virtual AFR sensor as a practical solution to be used in control systems AFR SI engine. The block diagram-based virtual MVEM AFR sensor presented in Fig. 5. There are two output models, the AFR and the manifold absolute pressure (MAP). However, these studies only focus on discussing the AFR.

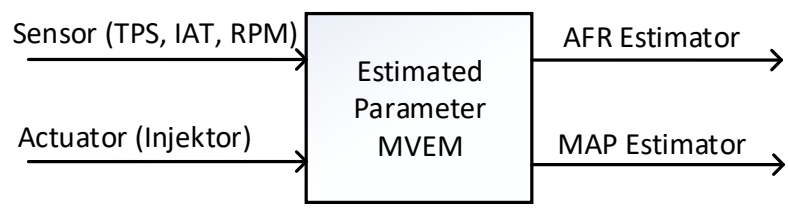

Fig. 5 Estimator AFR of open-loop or a so-called virtual AFR sensor

MVEM is a model as well as open-loop observer predicting variables on SI engines by making an average of each part of the dynamics of events SI engine. This model is physically based, very compact, and can be used in diesel and SI engines, with and without turbochargers [8].

Research on the observer or estimator based MVEM AFR has been performed using the method of maximum likelihood estimation and the Kalman filter to identify the parameters of the MVEM that cannot be calculated in the conventional [9]. Then, it is proceeding with the study of the observer to the SI engine for using the AFR control for correcting errors EKF 
state [10]. In the literature, it is explained that the study did not observe AFR directly, but by observing a multitude of sensors, using Constant Gain Extended Kalman Filter (CGEKF), to estimate the value of AFR. The sensor is TPS, crank shaft speed (n), MAP, mass air flow (MAFt), and MAFp. The AFR estimation is used to calculate the fuel in AFR control, which results in AFR = 14.7: $1 \pm 0.5$.

In another study, it is presented AFR virtual sensor-based neural network (NN) [11]. Results of the study are the observer open-loop with feedback sensors on SI engine to generate a new variable, namely Power $(\mathrm{kW})$, fuel pressure $(\mathrm{kPa})$, lambda (0.3--1.3, rms: 0.0232), HC (g/s), CO (g/s), $\mathrm{CO} 2(\mathrm{~g} / \mathrm{s})$ and NOx $(\mathrm{g} / \mathrm{s})$. Other studies on AFR observerbased $\mathrm{NN}$, it has also been done by using artificial neural network (ANN) feedforward, ANN recurrent neural networks (RNNs), MultiSpread-probabilistic neural networks (PNN), RBF-PNN, wavenet (wavelet NN), and Elman NN [1], [12]-[18]. Furthermore, it also included in the literature that another method that is not based on $\mathrm{NN}$ which is called support vector machine (SVM).

Another way to estimate the AFR is based on a pressure sensor in the cylinder SI engine [19]--[21]. It is also to create a new model, which uses methods of Linear Parametervarying (LPV) [22] and the new model to estimate the AFR by improving transport delay [23].

In this paper, the MVEM is selected to estimate the AFR, because it also considers transport delay to estimate the variables of the AFR that cannot be measured directly, with the variables of the AFR estimated to be in the combustion chamber, in contrast to the estimator AFR-type black-box, for example, NN. Although the accuracy of the NN method is high, this model does not consider the transport delay, because the models created cannot be known its dynamics, the process of its training is only based on the data set input and the target. Besides that, the MVEM has a compact form of mathematics and it has been tested from the 1990s to the present with a number of research in the field of SI engine injection control system that uses MVEM-based observer.

\section{STAGES OF RESEARCH, EXPERIMENTAL SETUP, AND DATA RETRIEVAL}

\section{A. Stages Research}

Generally, the stages of the research conducted are divided into four, namely the experimental setup and measurement, choosing the model structure, parameter estimation, and validation of the simulation as shown in Fig. 6.

\section{B. Experimental settings and measurement data}

Experiment data sets were obtained from direct measurements in real time on SI engine, a four-step Hyundai Verna (Accent LC) in 2002, G4EB, SOHC, $1500 \mathrm{cc}$. The plant was given additional types of UEGO lambda sensor, LM-2 Digital Air/Fuel Ratio Meter of Innovate Motorsports production, installed in the exhaust. UEGO sensor was selected because it had an area of the output wide linear sensor to changing conditions, in contrast to the type of sensor Bego, called lambda ON-OFF, which has a narrow linear area.

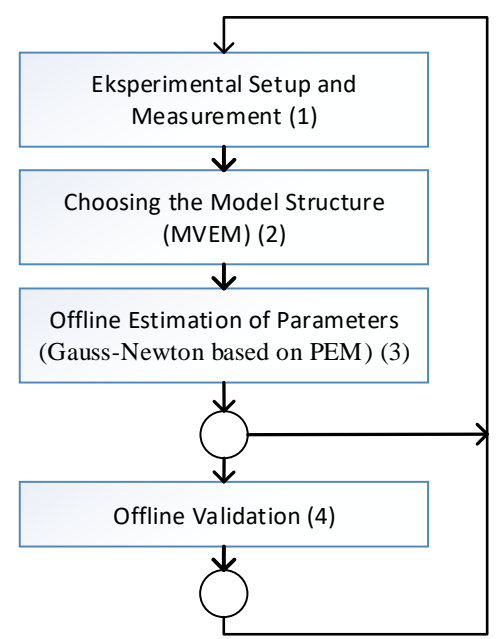

Fig. 6 Stages of research.

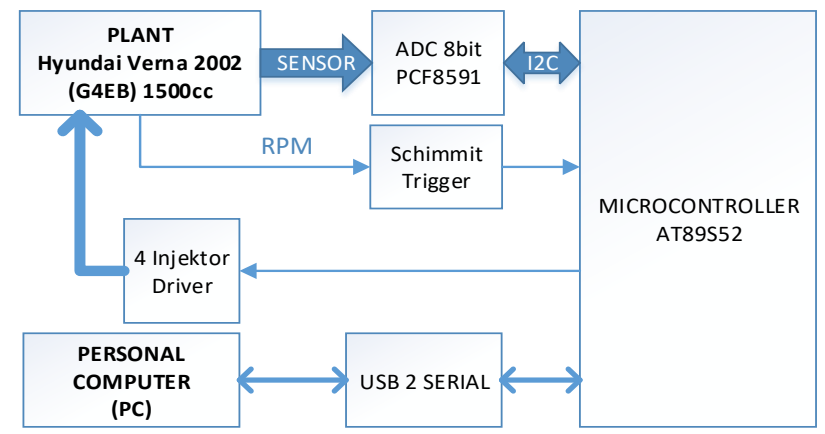

Fig. 7 A diagram of the circuit hardware for data real-time retrieval.

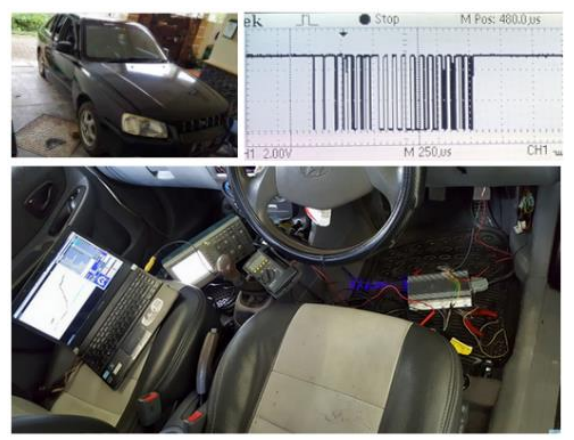

Fig. 8 Experimental settings for data retrieval.

Data acquisition process is performed to record data using a series data logger of microcontroller based connected to the software on the computer as presented in Fig. 7. Recorded data are the sensor output TPS, IAT, UEGO, the speed (n), and signal injector, assuming that the SI engine has reached the point working temperature of $80^{\circ}$ Celsius. Fig. 8 shows the experimental setup for data retrieval.

\section{Dynamics of MeAn VAlue Engine Model (MVEM)}

MVEM is one of SI engine injection systems pioneered and developed by Hendrick presented in the form of mathematics that is compact and can be customized parameters for different SI engines [24]--[26]. MVEM has been widely adopted in research on the SI engine involving control 
estimator-based/observer or estimator/observer alone [1], [17], [27]--[29]. MVEM is broadly divided into four subsystems, namely:

- the dynamics of the intake manifold,

- the dynamics of the mass flow rate of fuel,

- the engine rotation speed dynamics, and

- the dynamics of the air-fuel ratio (AFR).

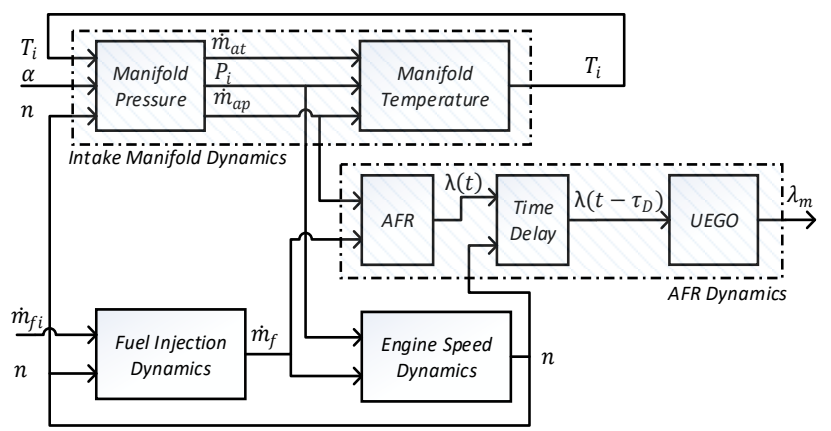

Fig. 9 General MVEM block diagram [1].

MVEM general block diagram is presented in Fig. 9 and is described further on exposure under.

\section{A. The Dynamics of Intake Manifold}

Dynamics of the intake manifold were analyzed based on the conservation of air mass into the intake manifold, consisting of two nonlinear differential equations that describe the manifold pressure $P_{i}$ and manifold temperature $T_{i}$. Manifold pressure $P_{i}$ is associated with the mass flow rate of water into the cylinder $\dot{m}_{a p}$, water mass flow rate past the throttle plate mat, manifold temperature $\mathrm{Ti}$, and atmosphere pressure as presented in a differential equation [3].

$$
\dot{P}_{l}=\left(R T_{i} / V_{i}\right)\left(-\dot{m}_{a p}+\dot{m}_{a t}\right)
$$

From (1), it can be seen that the dynamics of the air mass flow in the intake manifold which consists of two parts, the first is the air mass flow rate past the throttle plate $\dot{m}_{a t}$, which is associated with the throttle opening angle $\alpha$, and manifold pressure $\mathrm{P}_{\mathrm{i}}$, are presented in the following equation [3].

$$
\dot{m}_{a t}\left(\alpha, P_{i}\right)=m_{a t 1} P_{a} /\left(\sqrt{T_{a}}\right) \beta_{1}(\alpha) \beta_{2}\left(P_{i}\right)+m_{a t 0}
$$

with,

$$
\begin{aligned}
& \beta_{1}(\alpha)=1-\alpha_{1} \cos (\alpha)-\alpha_{2} \cos ^{2}(\alpha) \\
& P_{r}=P_{i} / P_{a} \\
& \beta_{2}\left(P_{r}\right)=\left(\sqrt{1-P_{r}^{P_{c 0}}}\right)\left(1+P_{c 1} P_{i}+P_{c 2} P_{i}^{2}\right)
\end{aligned}
$$

with $\alpha_{1}, \alpha_{2}, m_{a t 0}, m_{a t 1}, P_{c}$ is a constant with (5) is an equation that is different from the literature, which is the result of the modification, and the second is the rate of mass air flow of air into the cylinder (combustion chamber) $\dot{m}_{a p}$ associated with temperature manifold $T_{i}$, the pressure manifold $P_{i}$, and the engine rotation $n$, are presented in the following equation [3].

$$
\dot{m}_{a p}\left(n, P_{i}\right)=V_{d} /\left(120 R T_{i}\right)\left(e V P_{i}\right) n
$$

with $\mathrm{eV}$ is the volumetric efficiency and $\left(e V P_{i}\right)$ is the air charge normalized,

$$
\left(e V P_{i}\right)=s_{i}(n) P_{i}+y_{i}(n)
$$

with $s_{i}(n)$ and $y_{i}(n)$ is a positive function that depends on the engine speed $n$, approximated by a polynomial equation of order 3 ,

$$
\begin{aligned}
& s_{i}(n)=s_{i 0}+s_{i 1} n+s_{i 2} n^{2}+s_{i 3} n^{3} \\
& y_{i}(n)=y_{i 0}+y_{i 1} n+y_{i 2} n^{2}+y_{i 3} n^{3}
\end{aligned}
$$

with $s_{i 0}, s_{i 1}, s_{i 2}, s_{i 3}, y_{i 0} y_{i 1} y_{i 2} y_{i 3}$ is a constant.

In this paper, the temperature of the manifold $T_{i}$ is obtained directly from the plant as model input, so that the temperature dynamics of the manifold is ignored.

\section{B. Engine Speed Dynamics}

In this paper, the engine speed $n$ is obtained directly from the plant as model input, so that the engine rotation speed dynamics is ignored. Representation dynamics into differential equations presented in [1].

\section{Fuel Injection Dynamics}

Equations that describe the fuel mass flow rate of into the cylinder (combustion chamber), taking into account the evaporation of fuel in the intake manifold, and there are some that stick and flow through the channel walls of the intake manifold, stated as follows [1].

$$
\begin{aligned}
& \dot{m}_{f}=\dot{m}_{f v}+\dot{m}_{f f} \\
& \dot{m}_{f v}=\left(1-X_{f}\right) \dot{m}_{f i} \\
& \ddot{m}_{f f}=\left(1 / \tau_{f}\right)\left(-\dot{m}_{f f}+X_{f} \dot{m}_{f i}\right)
\end{aligned}
$$

with

$$
\begin{gathered}
X_{f}=X_{f 0}+X_{f 1} \dot{m}_{a p}\left(n, P_{i}\right)+X_{f 2} n+\ldots \\
\ldots+X_{f 3} P_{i}+X_{f 4} n^{2}+X_{f 5} P_{i}{ }^{2}
\end{gathered}
$$

with $X_{f 0}, X_{f 1}, X_{f 2}, X_{f 3}, X_{f 4}$, dan $X_{f 5}$ is a constant, $\mathrm{mfv}$ is the fuel mass flow which evaporates and into the cylinder (combustion chamber), $\dot{m}_{f f}$ is fuel the film mass flow attached to and flowing through the manifold wall, $\tau_{f}$ is fuel evaporation time constant, and $X_{f}$ is a fraction of the fuel film that produces how much fuel flowing in the walls of the manifold that depends on the operating point. Price $X_{f}$ ranges from 0.1 up to 0.5 , while $T_{f}$ ranges from 0:25 till 0.75 , both of which are affected the value of $P_{i}$ and $n$ [3]. Equation (13) is modified from the original equation [3] to obtain more precise results by increasing the polynomial order into a second order for the variable $n$ and $P_{i}$.

Variable input $\dot{m}_{f i}$ obtained from the signal is supplied to the injector, in milliseconds, taking into account the engine speed $\mathrm{n}$, injector flow rate $(\eta=234 \mathrm{cc} / \mathrm{min})$, and density $(\rho)$ 
fuel pertamax $\left(715-770 \mathrm{~kg} / \mathrm{m}^{3}\right)$, taken the average value, ie $\rho=(715+770) / 2=742,5 . \dot{m}_{f i}$ presented in the following equation.

$$
\dot{m}_{f i}=\rho \eta X_{\text {in }}(n / 2)
$$

with $X_{\text {in }}$ is the signal injector in milliseconds, shaped pulse $\mathrm{ON}$ and OFF the section which in the ON course that is noted.

\section{Dynamics of Air-Fuel Ratio (AFR):}

AFR is calculated by comparing the normalized air mass flow rate into cilynder $\dot{m}_{a p}$ and multiplication of numbers stekiometri $L_{t h}=14.7$ with the cylinder port fuel flow $\dot{m}_{f}$, as presented in (15).

$$
\lambda=\dot{m}_{a p} /\left(L_{t h} \dot{m}_{f}\right)
$$

Sensor $\lambda$ type of UEGO have a response in the area of the linear width of the representation of the condition of AFR good to poor condition $(\lambda>1)$, rich $(\lambda<1)$, or ideal (stoichiometric). This sensor is approached by the first-order system, presented in (16).

$$
\Delta_{m}(s) / \Delta_{\text {exh }}(s)=1 /\left(s \tau_{\lambda}+1\right)
$$

with $\Delta_{m}(s)$ and $\Delta_{e x h}(s)$ is the Laplace transform of $\lambda_{m}(t)$ and $\lambda_{\text {exh }}(t)$. Variable $\lambda_{m}$ is value $\lambda$ the measured of UEGO sensor, $\lambda_{\text {exh }}$ represents the value of $\lambda$ that will be read by the sensor, and $\tau_{\lambda}$ is the time constant of the sensor is temperature dependent on the exhaust pipe. The relationship between $\lambda_{\text {exh }}$ and $\lambda$, follow the following equation.

$$
\Delta_{\text {exh }}(s)=e^{-\tau_{d}} \Delta(s)
$$

with $\Delta(s)$ is the Laplace transform of $\lambda(t)$ and $\tau_{d}$ is the time transport delay between $\lambda(\mathrm{t})$ with $\lambda_{\text {exh }}(t)$. With inversed transform (16) and (17), obtained with the system of firstorder differential function as shown below.

$$
\begin{aligned}
& \dot{\lambda}_{m}=\left(1 / \tau_{\lambda}\right)\left(-\lambda_{m}+-\lambda_{\text {exh }}\right) \\
& \lambda_{\text {exh }}(t)=\lambda\left(t-\tau_{d}\right) .
\end{aligned}
$$

\section{E. Plant SI Engine}

Model of engine is approached by a system of multiple input multiple output (MIMO), has four inputs $\left(\dot{m}_{f i}, \alpha, n\right.$ and $\left.T_{i}\right)$, three states $\left(\dot{m}_{f f}, P_{i}\right.$ dan $\left.\lambda_{m}\right)$, and two outputs $\left(P_{i}\right.$ and $\left.\lambda_{m}\right)$. The equation for the states of $x_{1}, x_{2}$ dan $x_{3}$ are presented as

$$
\begin{gathered}
\dot{x}_{1}=\left(1 / \tau_{f}\right)\left(-x_{1}+X_{f}\left(x_{1}, u_{3}\right) u_{1}\right) \\
\dot{x_{2}}=R u_{4} / V_{i}\left(-\dot{m}_{a p}\left(u_{3}, u_{4}, x_{2}\right)+\dot{m}_{a t}\left(u_{2}, x_{2}\right)\right) \\
\dot{x_{3}}=\left(1 / \tau_{\lambda}\right)\left(-x_{3}+\lambda_{\text {exh }}\left(u_{1}, u_{3}, x_{1}, x_{2}\right)\right) .
\end{gathered}
$$

Output equation that depends on the states served as

$$
\begin{aligned}
& y_{1}=P_{i}=x_{2} \\
& y_{2}=\lambda=x_{2}
\end{aligned}
$$

with

$$
\dot{m}_{f}=x_{1}+\left(1-X_{f}\right) u_{1} \text {. }
$$

\section{MOdEls NONLINEAR GRAY-BoX (IDNLGREY)}

Modeling of gray-box is useful if it is known the relationship between the variables, constraints in the behavior of the model, or equation that explicitly represent the dynamics of the system. It can be performed using the system identification toolbox (MATLAB) to obtain the unknown parameters. Toolbox can be used for linear and nonlinear systems, discrete and continuous. However, because the laws of physics are expressed with continuous time, it is easier to construct models to the time domain continuous rather than discrete time domain [30].

The system to be modelled needs to be represented in the first-order nonlinear differential equations, which are generally presented in the following equation [30].

$\dot{x}(t)=F(t, x(t), u(t), \operatorname{par} 1, \operatorname{par} 2, \operatorname{par} 3, \ldots, \operatorname{par} N)$

$\dot{y}(t)=H(t, x(t), u(t), \operatorname{par} 1, \operatorname{par} 2, \operatorname{par} 3, \ldots, \operatorname{par} N)+e(t)$

$x(0)=x 0$

with $\dot{x}(t)=d x(t) / d t$ for continuous time representation. $F$ and $H$ is a linear or nonlinear function with a number of component parameter $N_{x}$ dan $N_{y}, N_{x}$ is the number of state and $N_{y}$ is the number of outputs.

ODE equation is expressed by the $\mathrm{C}$ language system resulting in a new format $\mathrm{C}$-MEX. Computing using format $\mathrm{C}$ MEXfiles is faster than the computation process $\mathrm{m}$ MEXformat file which is a file standardMATLAB. CMEXfile can be called by the main program written in the $\mathrm{m}$ file, it is required the process of compiling the C-MEX file to file new extension "mexw64". This conversion process requires a compiler from the outside named visual $\mathrm{C}++$. ODE equations are already tangible system file mexw64 is called by the main program in the $\mathrm{m}$-file to the learning process MVEM parameter is identified.

\section{PARAMETER ESTIMATION PREDICTION-BASED ERROR MINIMIZATION (PEM)}

\section{A. Prediction Error Minimization (PEM)}

Model the candidate $\mu^{*}$ that represents systems and data sets experimental $Z^{N}$ is defined by the following equations [31].

$$
\begin{aligned}
& \mu^{*}=\left\{\mu(\theta) \mid \theta \in D_{\mu}\right\} \\
& Z^{N}=[y(1), u(1), y(2), u(2), \ldots, y(N), u(N)]
\end{aligned}
$$

where $N$ is the number of pairs of input and output data sets, and $\theta$ is the vector of the model parameters. Vector estimation process model parameter $\theta$, of course, $\mu(\hat{\theta})$ is defined to be $\mu^{*}$, is accomplished by mapping $Z^{N}$ into $D_{\mu}$, and is defined by the following equation [31].

$$
Z^{N} \rightarrow \hat{\theta}_{N} \in D_{\mu}
$$

with all input-output pairs of $Z$, the number of $\mathrm{N}$ is mapped to the $\mathrm{D} \mu$ by the model $\mu$ with the estimated parameter vector $\hat{\theta}$. 
The mapping process is done by a certain parameter estimation method.

By using the estimated parameter vector $\hat{\theta}$, models $\mu$ is able to predict the output $y(t)$ of the system, with $\hat{y}(t)$ is the prediction of the model output $\mu(\hat{\theta})$, so that the error prediction is defined by the following equation [31].

$$
e(t, \theta)=y(t)-\hat{y}(t \mid \theta)
$$

with a model is stated to be good if it generates an error prediction $e(t, \theta)$ that is small or close to zero, and this requires a criterion as a function of the predictor. To get the criterion function $R(\theta)$ which is scalar, it is defined $V_{N}\left(\theta, Z^{N}\right)[31]$.

$$
V_{N}\left(\theta, Z^{N}\right)=\frac{1}{N} \sum_{t=1}^{N} R\left(e_{f}(t, \theta)\right) .
$$

Issues into a model parameter estimation process optimization become problems that require minimization criteria function, as defined by the following equation [31].

$$
\hat{\theta}_{N}=\arg \min V_{N}\left(\theta, Z^{N}\right) .
$$

The minimization method is known as the prediction-error minimization (PEM). From these explanations, the process of minimization based PEM can be summarized into the threestep process to estimate model parameters, namely choosing the model structure (gray-box, black-box, or other), select the function criteria (defined by function $R(\theta)$ which is scalar), and to minimize function criteria.

Function criterion $R_{N}(\theta)$ is selected for PEM-based applications that depends on matrix of the covariance of error prediction $e(t, \theta)$ presented as follows [31].

$$
R_{N}(\theta)=\frac{1}{N} \sum_{t=1}^{N} e(t, \theta) e^{T}(t, \theta)
$$

assuming that the error prediction $e(t, \theta)$ Gaussian distributed. Function criterion $R_{N}(\theta)$ should be scalar. Error predictions can be used directly as a function of criteria only if $e(t, \theta)$ is scalar-valued, that is when a system has a single output. In case the system has more than one output, it needs to make the criterion function $R_{N}(\theta)$ to be a scalar-valued. The function is defined generally to function as presented below [31].

$$
h\left(R_{N}(\theta)\right)=\operatorname{det}\left(R_{N}(\theta)\right) .
$$

Choice of the function criteria is considered to be statistically optimal statistical because it leads to maximum likelihood of errors Gaussian distributed prediction [32].

To minimize the criterion function, it is selected GaussNewton method, known as a rapid convergence process. The Gauss-Newton method uses routine numerically known as the Newton-Raphson algorithm. The step-by-step Gauss-Newton method has been described in previous studies [33].

\section{B. Strategy Experiments when Training Parameters MVEM}

There are 31 parameters in MVEM consisting of six parameters remain without estimation, namely $R, V_{d}, V_{i}, T_{a}$, $P_{a}$, and $L_{t h}$ and also 25 free parameters to be estimated, namely $\quad\left[\alpha_{1} ; \alpha_{2}\right], \quad\left[P_{c 0} ; P_{c 1} ; P_{c 2}\right], \quad\left[\dot{m}_{a t 0} ; \dot{m}_{a t 1}\right]$, $\left[s_{i 0} ; s_{i 1} ; s_{i 2} ; s_{i 3}\right], \quad\left[y_{i 0} ; y_{i 1} ; \quad y_{i 2} ; y_{i 3}\right], \quad\left[T_{f 0}\right]$, $\left[X_{f 0} ; X_{f 1} ; X_{f 2} ; X_{f 3} ; X_{f 4} ; X_{f 5}\right], \quad\left[\tau_{d 0}, \tau_{d 1}\right]$, dan $\left[\tau_{\lambda}\right]$. $\left[s_{i 0} ; s_{i 1} ; s_{i 2} ; s_{i 3}\right],\left[y_{i 0} ; y_{i 1} ; y_{i 2} ; y_{i 3}\right]$.

Classification of free parameters that influence the dynamics of each system is described in Fig. 10.

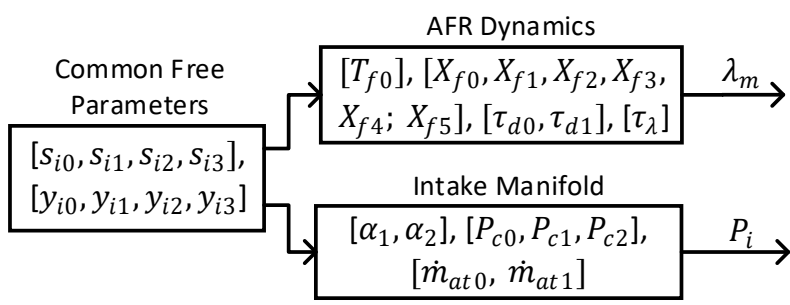

Fig. 10 Free parameters influencing the dynamics of the system.

Free parameters together, that $\left[s_{i 0} ; s_{i 1} ; s_{i 2} ; s_{i 3}\right]$, $\left[y_{i 0} ; y_{i 1} ; y_{i 2} ; y_{i 3}\right]$, influence the dynamics of the AFR and the intake manifold. Free parameters, which only affect the dynamics of AFR are $\left[T_{f 0}\right],\left[X_{f 0} ; X_{f 1} ; X_{f 2} ; X_{f 3} ; X_{f 4} ; X_{f 5}\right]$, $\left[\tau_{d 0}, \tau_{d 1}\right]$, and $\left[\tau_{\lambda}\right]$, [whereas the free parameters, which only affect the dynamics of the intake manifold are $\left[\alpha_{1} ; \alpha_{2}\right]$, $\left[P_{c 0} ; P_{c 1} ; P_{c 2}\right]$, and $\left[\dot{m}_{a t 0} ; \dot{m}_{a t 1}\right]$.

There are three stages of training or learning done, the first process training for AFR dynamics, the second is the process training for the dynamics of the intake manifold, and the third is the process training by combining the dynamics of the AFR and the intake manifold in a large system model. This is done to minimize the computational process.

In the first stage, it is assumed model does not require the estimation of MAP /Pi close-loop,as presented in Fig. 11, with a value of Pi directly obtained from the MAP sensor. From here it can be seen that the model is a MISO system with five inputs $=\left[\begin{array}{lllll}u_{1} & u_{2} & u_{3} & u_{4} & u_{5}\end{array}\right]^{T}=\left[\begin{array}{l}\dot{m}_{f i} \alpha n T_{i} P_{i}\end{array}\right]^{T}$, $x=\left[\begin{array}{ll}x_{1} & x_{2}\end{array}\right]^{T}=\left[\begin{array}{ll}\dot{m}_{f f} & \lambda_{\text {new }}\end{array}\right]^{T}$, and one output $y=\left[y_{1}\right]=$ $\left[\lambda_{\text {new }}\right]$.

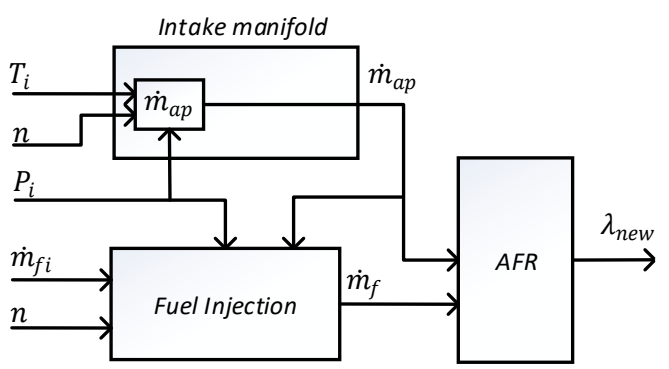

Fig. 11 MVEM block for identification strategy in the first stage.

Target data $\lambda_{\text {new }}=\lambda_{m}\left(t+\tau_{D}\right)$ are obtained from experimental data sets of the UEGO sensor shifted forward as far as $\tau_{D}=f(n)=\tau_{d 0}+\tau_{d 1} n$. The new variable $\lambda_{\text {new }}$ is needed because it is not found a way to build a non-linear model of grey-box functions delay into a syntax that is necessary toolbox, or toolbox in MATLAB that does not have this facility, so it requires manually one by one to see the effect of $\tau \mathrm{D}$ for the accuracy of the results parameter estimation, by trying to enter $5 \times 6=30$ points. 
Free parameters which influence the dynamics of AFR are $\left[T_{f 0}\right],\left[X_{f 0} ; X_{f 1} ; X_{f 2} ; X_{f 3} ; X_{f 4} ; X_{f 5}\right],\left[\tau_{d 0}, \tau_{d 1}\right]$, dan $\left[\tau_{\lambda}\right]$ coupled with the free parameters together, that $\left[s_{i 0} ; s_{i 1} ; s_{i 2} ; s_{i 3}\right]$ and $\left[y_{i 0} ; y_{i 1} ; y_{i 2} ; y_{i 3}\right]$. Parameters $\left[\tau_{d 0}, \tau_{d 1}\right]$ affect sthe transport delay $\tau_{D}$. The process training using the Gauss-Newton method based PEM is done by inserting a 30 point $\tau_{D}$ one by one manually. Estimated free parameters $\left[\hat{\tau}_{d 0}, \hat{\tau}_{d 1}\right]$ can be obtained from the condition normalized root mean square error (NRMSE) and to determine the smallest done manually by describing the shape of its surface and contour. More specifically, it is discussed further in the "Results and Discussion". While other estimated free parameters are obtained automatically from the process training using the Gauss-Newton-based PEM, namely $\left[\widehat{T}_{f 0}\right]$, $\left[\hat{X}_{f 0} ; \hat{X}_{f 1} ; \hat{X}_{f 2} ; \hat{X}_{f 3} ; \hat{X}_{f 4} ; \hat{X}_{f 5}\right],\left[\hat{\tau}_{\lambda}\right]$, added by the free parameters of $\left[\hat{s}_{i 0} ; \hat{s}_{i 1} ; \hat{s}_{i 2} ; \hat{s}_{i 3}\right],\left[\hat{y}_{i 0} ; \hat{y}_{i 1} ; \hat{y}_{i 2} ; \hat{y}_{i 3}\right]$. And of course, the process training requires initialization.

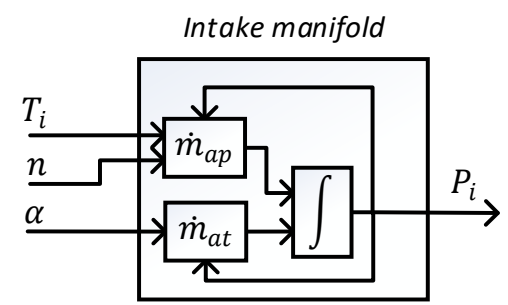

Fig. 12 MVEM block for identification strategy in the second stage.

In the second phase of the system formed the open-loop by $P_{i}$ as the output of the system, as in Fig. 12. From here, it can be seen that that the model is a MISO system with three inputs, namely $=\left[\begin{array}{lll}u_{1} & u_{2} & u_{3}\end{array}\right]^{T}=\left[\begin{array}{lll}\alpha & n T_{i}\end{array}\right]^{T}$, one state $x=$ $\left[x_{1}\right]^{T}=\left[P_{i}\right]$, and one output $y=\left[y_{1}\right]=\left[P_{i}\right]$. Target data $P_{i}$ is obtained from a data set of record results MAP sensor. Free parameters which influence the dynamics of the intake manifold is $\left[\alpha_{1} ; \alpha_{2}\right],\left[P_{c 0} ; P_{c 1} ; P_{c 2}\right]$, and $\left[\dot{m}_{a t 0} ; \dot{m}_{a t 1}\right]$, and added by a joint free parameters, namely $\left[s_{i 0} ; s_{i 1} ; s_{i 2} ; s_{i 3}\right]$ and $\left[y_{i 0} ; y_{i 1} ; y_{i 2} ; y_{i 3}\right]$. Early initialization parameters for shared freely are obtained from the first stage above and others made random. After the process of training with the Gauss-Newton method based PEM is completed, it is obtained estimated free parameters of $\left[\hat{\alpha}_{1} ; \hat{\alpha}_{2}\right],\left[\hat{P}_{c 0} ; \hat{P}_{c 1} ; \hat{P}_{c 2}\right]$, and $\left[\widehat{\dot{m}}_{a t 0} ; \widehat{\dot{m}}_{a t 1}\right]$, added by the free parameters of $\left[\hat{s}_{i 0} ; \hat{s}_{i 1} ; \hat{s}_{i 2} ; \hat{s}_{i 3}\right],\left[\hat{y}_{i 0} ; \hat{y}_{i 1} ; \hat{y}_{i 2} ; \hat{y}_{i 3}\right]$.

In the third stage, the two subsystems, namely the AFR and the dynamics, intake manifold combined into one is presented in Fig. 13. It appears that the model is a MIMO system with four inputs $=\left[\begin{array}{llll}u_{1} & u_{2} & u_{3} & u_{4}\end{array}\right]^{T}=\left[\begin{array}{ll}\dot{m}_{f i} \alpha n T_{i}\end{array}\right]^{T}$, three states $x=\left[\begin{array}{lll}x_{1} & x_{2} & x_{3}\end{array}\right]^{T}=\left[\dot{m}_{f f} P_{i} \lambda_{\text {new }}\right]^{T}$, and two outputs $y=\left[y_{1} y_{2}\right]=\left[\begin{array}{ll}P_{i} & \lambda_{\text {new }}\end{array}\right]$. Target data $P_{i}$ and $\lambda_{\text {new }}$ sensor data are obtained from MAP $/ \mathrm{Pi}$ and UEGO. Initialization beginning to use all the free parameter values obtained from the second stage above.

For the record, there are three files C-MEX generated for each of the above steps. Then, the process of the estimation of
MVEM parameters uses the Gauss-Newton based PEM method using the following syntax in m-file MATLAB.

“pem(z, nlgr, 'Display', 'Full', 'SearchMethod','gn')"

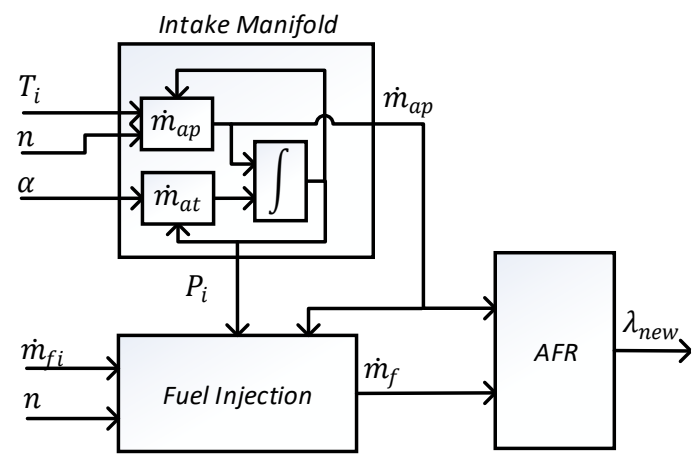

Fig. 13 MVEM block for identification strategy in the third stage.

\section{RESULTS AND DISCUSSION}

This section discusses the analysis, research data, transport delay $\tau_{D}$ on AFR, validation of results, and analysis. The success rate is obtained by calculating the root mean square error (RMSE) and NRMSE, with notes saturation value of UEGO sensor, so as to calculate its error criteria, the outputs of the model are also given the function of saturation of $\lambda \varepsilon$ $[0.685,1.285]$.

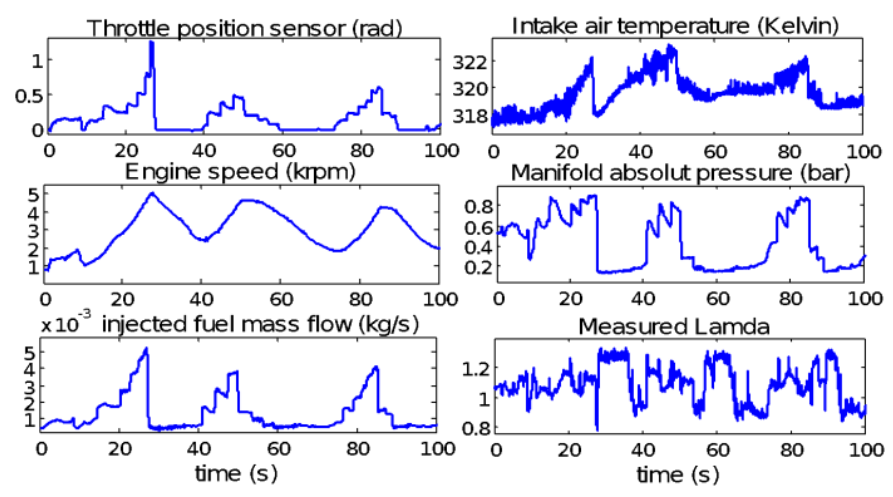

Fig. 14 Data set of the first validation with condition gear 1 to 2 .

\section{A. Research Data}

There are three kinds of data set that are used with each dataset of more than 10,000 pairs of input and output with time of sampling 0:01 seconds. The first set of data are used for process training or learning when MVEM parameter identification. Data set of training were taken by positioning gear (gear) one, then moved into high gear (gear) two, and finally to the gear three. The last two data sets were used for validation, called data validation sets, used to assess the success of the model estimator AFR. In contrast to the method of the collection of the data set training, data sets of validation were performed in two ways:

1. the data set of validation 1 were taken by positioning gear (gear) one, then moved into gear two, and

2. the data set of validation 2 were taken by positioning gear (gear) one, then moved directly into gear three. 
For injector, it was given a certain amount of signal pulse when the injector should be ON. As for the gas pedal, played by the driver while running the car on the highway. In this section, it is presented one example of a data set of studies, the data set of the first validation as presented in Fig. 14.

\section{B. Results Identification MVEM}

Process training produces estimated parameters, namely (1) free parameters which only affect the AFR dynamics or dynamics of the intake manifold only, and (2) along with free parameters that affect the dynamics of the AFR and the intake manifold. The free parameters are presented in Table I and the fixed parameters are presented in Table II.

TABLE I

I-PARAMETER PARAMETER VALUE FREE MVEM RESULTS IDENTIFICATION

\begin{tabular}{|c|c|c|c|}
\hline Parameter & Value & Parameter & Value \\
\hline$\hat{\alpha}_{1}$ & 0.96408 & $\hat{y}_{i 2}$ & 108.64 \\
\hline$\hat{\alpha}_{2}$ & $-5.4292 \mathrm{e}-2$ & $\hat{y}_{i 3}$ & -8.3926 \\
\hline$\hat{P}_{c 0}$ & 0.27939 & $\widehat{T}_{f}$ & 0.1554 \\
\hline$\hat{P}_{c 1}$ & 0.28459 & $\hat{X}_{f 0}$ & 3.0906 \\
\hline$\hat{P}_{c 2}$ & 1.73280 & $\hat{X}_{f 1}$ & 52.566 \\
\hline$\hat{\dot{m}}_{a t 0}$ & -0.08776 & $\hat{X}_{f 2}$ & -0.7243 \\
\hline$\hat{\dot{m}}_{a t 1}$ & 28.5710 & $\hat{X}_{f 3}$ & -4.8022 \\
\hline$\hat{s}_{i 0}$ & 385.190 & $\hat{X}_{f 4}$ & 0.0110 \\
\hline$\hat{s}_{i 1}$ & 1088.8 & $\hat{X}_{f 5}$ & 1.5280 \\
\hline$\hat{s}_{i 2}$ & -358.90 & $\hat{\tau}_{d 0}$ & 0.6140 \\
\hline$\hat{s}_{i 3}$ & 35.042 & $\hat{\tau}_{d 1}$ & -0.0940 \\
\hline$\hat{y}_{i 0}$ & 252.49 & $\hat{\tau}_{\lambda}$ & 0.0555 \\
\hline$\hat{y}_{i 1}$ & -347.67 & $\mathrm{X}_{1}$ & $\mathrm{X}$ \\
\hline
\end{tabular}

TABLE II

VALUE PARAMETER-PARAMETER FIXED MVEM

\begin{tabular}{|c|c|c|c|}
\hline Parameter & Value & Parameter & Value \\
\hline$R$ & 0.00287 & $L_{t h}$ & 14.7000 \\
\hline$V_{d}$ & 0.00150 & $T_{a}$ & 301.000 \\
\hline$V_{i}$ & 0.00170 & $P_{a}$ & 0.95580 \\
\hline
\end{tabular}

\section{Transport Delay $\tau D$ on AFR}

An experiment to get the value of $\tau_{d 0}$ and $\tau_{d 1}$ was done during the initial training MVEM parameter when identifying MVEM. The function of $\tau_{d}=f(n)$ is approximated linearly derived from the equation of a straight line with the two points known points of $\left(x_{1}, y_{1}\right)=\left(1, y_{1}\right)$ and $\left(x_{2}, y_{2}\right)=\left(6, y_{2}\right)$, with $x=n$ in unit of krpm $y=\tau_{d}$ in seconds. By changing the value of $\mathrm{y} 1$ and $\mathrm{y} 2$ manually in every process of training when making MVEM parameter identification with the first phase of the strategy (described in Section "Parameters Training Strategy Experiments currently MVEM" above), then the result is NRMSE value. By combining $y_{1}$ dan $y_{2}, y_{1}=$ $0.25 \mathrm{~s} ; 0.35 \mathrm{~s} ; 0.40 \mathrm{~s} ; 0.45 \mathrm{~s} ; \mathrm{S} 0.50,0.55 \mathrm{~s}$ and $y_{2}=0.00 \mathrm{~s}$; $0.10 \mathrm{~s} ; 0.15 \mathrm{~s} ; 0.20 \mathrm{~s} ; 12.25 \mathrm{~s}$, the obtained NRMSE value is 6 x $5=30$ points.

Furthermore, from the 30 pairs of data input $\left(y_{1}, y_{2}\right)$ and output $z=$ NRMSE, it is approximated by (32).

$$
\begin{aligned}
N R M S E=z=f & \left(y_{1}, y_{2}\right) \\
& =k_{1} y_{1}^{2}+k_{2} y_{2}^{2}+k_{3} y_{1} y_{2}+k_{4} y_{1} \\
& +k_{5} y_{2}+k_{6} .
\end{aligned}
$$

Using toolbox of MATLAB, "polyfitn" produced values of $k_{1}=6.5992 e-04, \quad k_{2}=8.2679 e-04, \quad k_{3}=0.0013$ $k_{4}=-7.4980 e-04, \quad k_{5}=-7.5654 e-04, \quad k_{6}=0.0898$. The shape of surface the equation is presented in Fig. 15 and the shape of its surface. It can be seen that the curve is concave with a certain minimum value. This minimum point is sought to produce estimates of transport delay $\tau_{d}$.

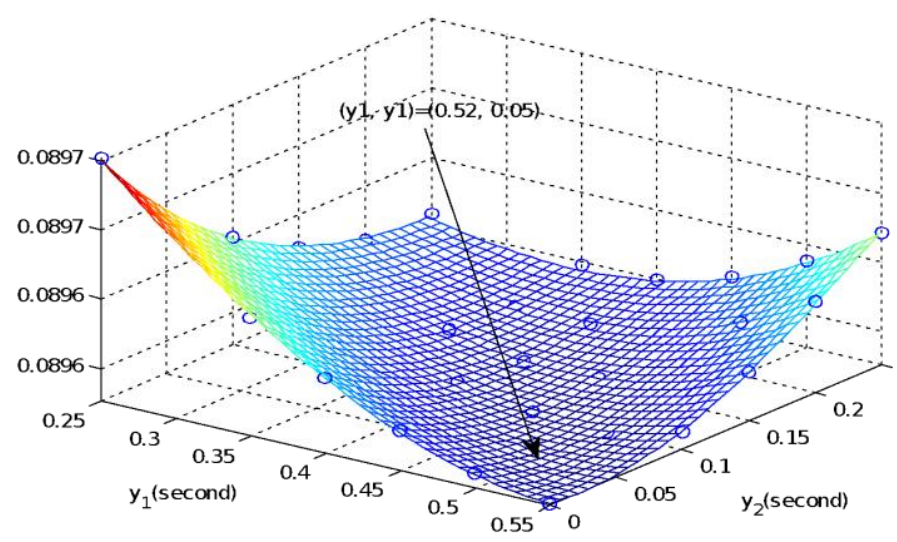

Fig. 15 Surface effect of variable $(y 1, y 2)$ in function of $\tau d=f(n)$ against NRMSE on MVEM identifier.

From shape, its contour as presented in Fig. 16 can be estimated that NRMSE is worth of minimum at $\left(\mathrm{y}_{1}, \mathrm{y}_{2}\right)=$ $\left(\mathrm{y}_{1, \mathrm{opt}}, \mathrm{y}_{2, \mathrm{opt}}\right)=(0.52,0.05)$ by substituting two points $\left(\mathrm{x}_{1}, \mathrm{y}_{1}\right)=\left(1, \mathrm{y}_{1, \mathrm{opt}}\right)=(1,0.52)$ and $\left(\mathrm{x}_{2}, \mathrm{y}_{2}\right)=\left(6, \mathrm{y}_{2, \mathrm{opt}}\right)=$ $(6,0.05)$ into the equation of the straight line given two points, then it is obtained transport delay $\tau_{\mathrm{d}}=f(n)=\tau_{\mathrm{d} 0}+$ $\tau_{\mathrm{d} 1} \mathrm{n}=-0.094 \mathrm{n}+0.614$.

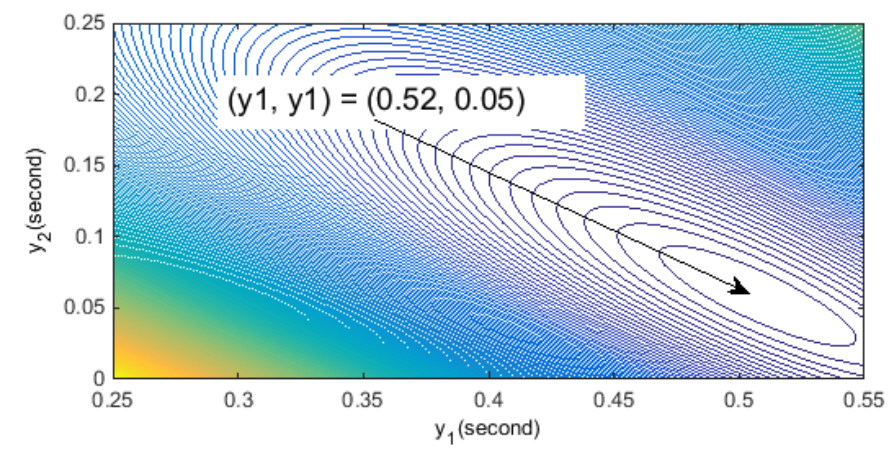

Fig. 16 Contour effect of variable $(y 1, y 2)$ in function of $\tau d=f(n)$ against NRMSE results of MVEM.

\section{Validation of Results}

Error criteria value obtained from the validation of estimator AFR the open-loop is satisfactory, with two data for validation generate NRMSE value below 0.1 , or $10 \%$, and the average RMSE $=0.07196$ and NRMSE $=0.06831$. Value criteria error and the average are presented in Table III. 
TABLE III

PARAMETER VALUES-PARAMETER NON MVEM RESULTS IDENTIFICATION

\begin{tabular}{|c|c|c|c|}
\hline Data set & Estimator & RMSE & NRMSE \\
\hline $1^{\text {st }}$ Validation & $\hat{\lambda}_{m}$ & 0.08221 & 0.07568 \\
\hline $2^{\text {nd }}$ Validation & $\hat{\lambda}_{m}$ & 0.06170 & 0.06094 \\
\hline \multicolumn{2}{|c|}{ Mean } & 0.07196 & 0.06831 \\
\hline
\end{tabular}

Pattern of the output signal MVEM, estimator $\hat{\lambda}_{m}$ indicating a trend that leads similar to the target data (measurement $\hat{\lambda}_{m}$ ). Graph error estimation and $\hat{\lambda}_{m}$ the first model presented in Fig. 17. There are certain points that lead to errors of great value, marked with a circle, which for certain conditions, the model still cannot represent the dynamics of the accurately plant, since the system is openloop so that no correction of the difference between $\hat{\lambda}_{m}$ and $\lambda_{m}$ to fix the state.
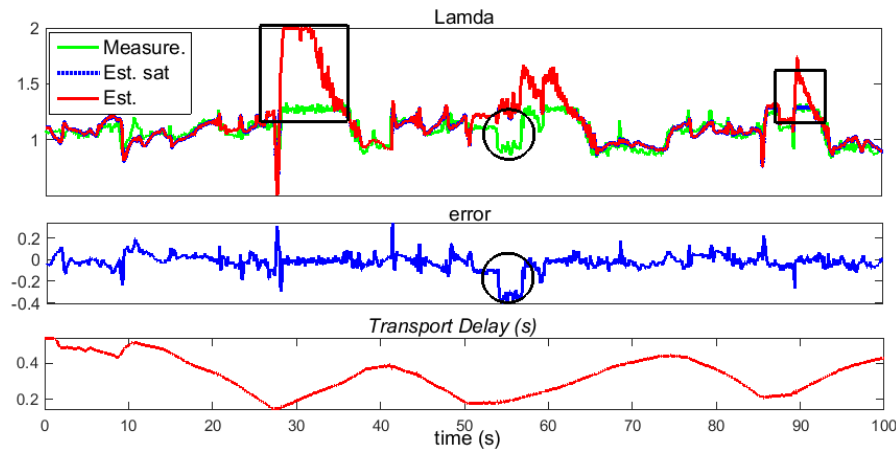

Fig. 17 Estimation graph $\hat{\lambda}_{m}$, error and transport delay using the first validation data.

While the area is placed inside the box indicates that the estimated $\hat{\lambda}_{m}$ is able to predict unmeasured values by measuring $\hat{\lambda}_{m}$, because of the limitations of measuring instruments (UEGO sensor) itself, with $\lambda \in[0.685,1.285]$.

\section{E. Research Findings}

The aim of this study is to develop models or algorithms for estimating the AFR in the cylinder (combustion chamber) SI engine without the effect of the transport delay. From the experiment, the delay transport is influenced by the engine speed $n(\mathrm{krpm})$, ie $\tau_{d}=(-0.094 n+0.614)$ seconds. Value criteria error (RMSE and NRMSE) can only be calculated if the actual target value is identified, not an estimate. In this case, the variable $\lambda$ can not be measured because it is in the state. Therefore, the error criterion, estimator $\hat{\lambda}$, is approached by calculating error estimators $\hat{\lambda}_{m}$ of the measurement. It is assumed that if the estimator model output $\hat{\lambda}_{m}$ produces a high success rate, it can be stated that the success rate in the variable estimation state (estimator $\lambda$ ) is also high where the value of $\hat{\lambda}_{m}$ is obtained from $\lambda$ delayed by transport delay $\tau_{d}$.Variable $\lambda_{m}$ represents normalized readable AFR UEGO sensor and $\lambda$ represents a normalized value of AFR in the combustion chamber.

It is presented a graph estimator $\hat{\lambda}$ displayed along with the measurement $\lambda_{m}$ of the first, second, and third with the use of validation data, presented in Fig. 18. Two graph on the right is the result of zooming the graph on the left in the circled area. From the graph, it appears that the estimator $\lambda$ move forward (faster) and is followed by a measurement signal $\lambda_{m}$. It can be seen more clearly in the transient conditions.

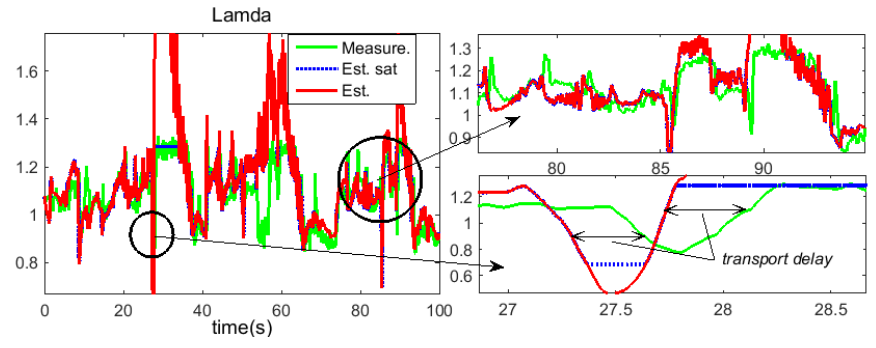

Fig. 18 Estimation Graph $\hat{\lambda}_{m}$, without delay to the measurement of the first model using the first validation data.

The graph in Fig. 18 shows the transport delay between estimators $\lambda$ and measurement $\lambda_{m}$. In accordance with previous discussion, with the transport delay, the sensor $\lambda_{m}$ can only be used as a feedback for control of "correction" AFR, not as a feedback for control of the "main" stand-alone AFR. With the development of the estimator algorithm $\hat{\lambda}$, the estimator $\hat{\lambda}$ without the effect of the transport delay is expected to be used as a feedback to the control system of the "main" stand-alone.

\section{F. Comparison with Other Research}

Until now, it has not been in the literature on previous studies that are identical to this current study. There are some publications that discuss the same issue regarding the estimation of the AFR, but the method, the approach of models, input systems, and data sets are different from this present research. Additionally previous studies use the same method for the estimation, but the estimated variable or system is different, and several studies even build a new model for estimating the AFR with the effect of transport delay or not.

Research that produces the highest success rate among other research using methods based on $\mathrm{NN} \mathrm{[1],} \mathrm{which} \mathrm{is} \mathrm{the}$ criterion error MRE $=0976 \%$, higher compared to this paper with NRMSE $=6,831 \%$ with a model estimator equally openloop (so called virtual AFR sensor). The difference is in the way research data collected. In previous research, data retrieval to obtain data set of studies were conducted by plant simulation, not plant actually [1]. And of course, the model (simulation) certainly has errors against the real plant, in which in that paper it is not explained the criteria for error model (simulation) itself.

\section{CONCLUSION}

The experiment produces estimator $\hat{\lambda}$ with a success rate that is calculated using criteria value of error estimation $\hat{\lambda}_{m}$ to the UEGO sensor measurement $\left(\lambda_{m}\right)$. The error criteria used are the RMSE and NRMSE, respectively, generated by their average value of 0.0720 and $0.0683=6.831 \%$. The result of experiment shows that the position signal $\hat{\lambda}$ is preceded $\hat{\lambda}_{m}$ in the time domain graph $t$ because it is influenced by the 
transport delay $\tau_{D}$ which the value changes influenced by engine rotation speed $n$, that is $\tau_{d}=(-0.094 n+0.614)$. The estimator AFR $(\hat{\lambda})$ generated needs to be tested in future studies as feedback in the control of the "main" AFR to obtain the output AFR accuracy and precision, both during conditions transient or steady state, both offline and online, where in the study discusses the special AFR control system, not on the estimated AFR.

\section{ACKNOWLEDGMENT}

Special thanks to the Bureau of Planning and International Affairs, Secretary General, Ministry of Education and Culture (Kemdikbud) of the Republic of Indonesia that has supported this research by providing Scholarship in 2015 to the author [1]

\section{REFERENCES}

[1] Y. Shi, D.-L. Yu, Y. Tian, and Y. Shi, "Air-Fuel Ratio Prediction and NMPC for SI Engines With Modified Volterra Model and RBF Network," Eng. Appl. Artif. Intell., Vol. 45, pp. 313-324, Oct. 2015.

[2] Tom Dentom, Automobile Electrical and Electronic System. Burlington, MA: Elsevier Butterworth-Heinemann, 2004.

[3] Christian Winge Vigild, Elbert Hendricks, and Spencer C Sorenson, "The Internal Combustion Engine Modelling: Modelling, Estimation and Control Issues," Technical University of Denmark, Lyngby, 2002.

[4] H. Tang, L. Weng, ZY Dong, and R. Yan, "Adaptive and Learning Control for SI Engine Model With Uncertainties," IEEEASME Trans. Mechatron, Vol. 14, No. 1, pp. 93-104, Feb. 2009.

[5] DG Copp, KJ Burnham, and FP Lockett, "Model Comparison for Feedforward Air/Fuel Ratio Control," Control '98. UKACC International Conference on (Conf. Publ. No. 455), 1998, Vol. 1, pp. 670-675.

[6] Toyota Computer Controlled System (TCCS), Toyota Technica Training, 1997.

[7] D. Marin, I. Hiticas, and L. Mihon, "Fuzzy Logic Control Applied on SI Engine Concerning the Injection Time Evolution," 2012 IEEE 13th International Symposium on Computational Intelligence and Informatics (CINTI), 2012, pp. 279-284.

[8] E. Hendricks and J. Luther, "Model and Observer Based Control of Internal Combustion Engines," Proceedings of the 1st International Workshop on Modeling Emissions and Control in Automotive Engines, MECA'01, 2001, pp. 9-20.

[9] H. Melgaard, E. Hendricks, and H. Madsen, "Continuous Identification of a Four-Stroke SI Engine," American Control Conference, 1990, 1990, pp. 1876-1881.

[10] E. Hendricks, J. Poulsen, MB Olsen, PB Jensen, M. Fons, and C. Jepsen, "Alternative Observers for SI Engine Air/Fuel Ratio Control," Proceedings of 35th IEEE Conference on Decision and Control, 1996, Vol. 3, pp. 2806-2811

[11] EL Hanzevack, TW Long, CM Atkinson, and M. Traver, "Virtual Sensors for Spark Ignition Engines Using Neural Networks," Proceedings of the 1997 American Control Conference, 1997, Vol. 1, pp. 669-673.

[12] SS Kamat, H. Javaherian, VV Diwanji, JG Smith, and KP Madhavan, "Virtual Air-Fuel Ratio Sensors for Engine Control and Diagnostics," 2006 American Control Conference, 2006, pp. 7

[13] X. Donghui, L. Yuelin, and Zhouzhe, "Study on Transient Air-Fuel Ratio Predictive Model of Gasoline Engine Based on Artificial Intelligence," 2014 7th International Conference on Intelligent Computation Technology and Automation, 2014, pp. 742-745.
[14] N. Cesario, M. Lavorgna, and F. Pirozzi, "Modelling On-Off Virtua Lambda Sensors Based on Multi-Spread Probabilistic Neural Networks," 10th IEEE Conference on Emerging Technologies and Factory Automation 2005 (ETFA 2005), 2005, Vol. 1, pp. 6

[15] L. Wu and JJ Liu, "Comparative Research Transient Air-Fuel Ratio Control Strategy Based on Fuzzy Control and Neural Network," Appl. Mech. Mater., Vol. 643, pp. 66-71, Sep. 2014.

[16] WK Yap and V. Karri, “ANN virtual sensors for emissions prediction and control," Appl. Energy, Vol. 88, No. 12, pp. 4505-4516, Dec. 2011.

[17] SW Wang, DL Yu, JB Gomm, GF Page, and SS Douglas, "Adaptive Neural Network Model Based Predictive Control for Air-Fuel Ratio of SI Engines," Eng. Appl. Artif. Intell., Vol. 19, No. 2, pp. 189-200, Mar. 2006.

[18] I. Arsie, C. Pianese, and M. Sorrentino, "A Procedure to Enhance Identification of Recurrent Neural Networks for Simulating Air-Fuel Ratio Dynamics in SI Engines," Eng. Appl. Artif. Intell., Vol. 19, No. 1 pp. 65-77, Feb. 2006.

[19] R. Pradhan, P. Ramkumar, and S. Suhan, "Estimation of Air-Fuel Ratio (AFR) in a Sark-Ignition (SI) Engine from Cylinder Pressure Measurements", IJRRAS, Vol. 13, No. 3, pp. 707-715, Dec. 2012.

[20] A. Yazdani et al., "Air Charge and Residual Gas Fraction Estimation for a Spark-Ignition Engine Using In-Cylinder Pressure," SAE Technical Paper, 2017.

[21] M. Kumar and T. Shen, "Estimation and Feedback Control of Air-Fuel Ratio for Gasoline Engines," Control Theory Technol., Vol. 13, No. 2 pp. 151-159, May 2015

[22] D. Efimov, S. Li, Y. Hu, S. Muldoon, H. Javaherian, and VO Nikiforov, "Application of Interval Observers to Estimation and Control of AirFuel Ratio in a Direct Injection Engine," American Control Conference 2015 (ACC), 2015, pp. 25-30.

[23] T. Laurain, Z. Lendek, J. Lauber, and RM Palhares, "A New Air-Fuel Ratio Model Fixing the Transport Delay: Validation and Control," 2017 IEEE Conference on Control Technology and Applications (CCTA), 2017, pp. 1904-1909.

[24] E. Hendricks and S. Sorenson, "Mean Value SI Engine Model for Control Studies," American Control Conference 1990, 1990, pp. 1882 1887.

[25] E. Hendricks and SC Sorenson, "SI Engine Controls and Mean Value Engine Modelling," SAE Technical Paper, 1991.

[26] E. Hendricks, "A Generic Mean Value Engine Model for Spark Ignition Engines," Proceedings of the 41st Simulation Conference, SIMS 2000 2000.

[27] J. Na, G. Herrmann, C. Rames, R. Burke, and C. Brace, "Air-Fuel-Ratio Control of Engine System with Unknown Input Observer," 2016 UKACC 11th International Conference on Control (CONTROL), 2016 pp. $1-6$.

[28] J. Espinoza-Jurado, E. Dávila, J. Rivera, JJ Raygoza-Panduro, and S Ortega, "Robust Control of the Air to Fuel Ratio in Spark Ignition Engines with Delayed Measurements from a UEGO Sensor," Math. Probl. Eng., Vol. 2015, pp. 1-13, 2015.

[29] M. Lei, Z. Chunnian, L. Hong, L. Jie, L. Wen, and L. Xianghua, "Research on Modeling and Simulation of SI Engine for AFR Contro Application," Biotechnol. Indian J., Vol. 10, No. 24, 2014.

[30] Lennart Ljung, "System Identification Toolbox ${ }^{\mathrm{TM}}$ User's Guide." The MathWorks, Inc, Sep-2012.

[31] F. Spuri and L. Goes, "Modeling and Parametric Identification of a Variable-Displacement Pressure-Compensated Pump," The 15th Scandinavian International Conference on Fluid Power, SICFP'17, 2017.

[32] Lennart Ljung, System Identification Theory for the User (Second Edition). Prentice Hall, 1999.

[33] A. Croeze, L. Pittman, and W. Reynolds, "Nonlinear Least-Squares Problems with the Gauss-Newton and Levenberg-Marquardt Methods,' Technical report, University of Mississipi, Department of Mathematics, June 2012 